\title{
CRECIMIENTO ECONÓMICO E INVERSIÓN PÚBLICA: UNA PANORÁMICA
}

\author{
Gaspar Núñez Rodríguez*
}

Centro de Estudios SocioEconómicos, Universidad Autónoma de Coahuila

(Recibido 26 de septiembre 2003, aceptado 15 de diciembre 2003)

\begin{abstract}
En este trabajo revisamos los principales modelos teóricos en los que se ha basado la investigación de la relación entre la inversión pública y el crecimiento económico, así como los resultados empíricos más característicos arrojados por la aplicación de dichos análisis. La literatura empírica revisada sugiere, crecientemente, que la mencionada relación existe, que es positiva y significativa, y que la inversión pública puede tener, en algunos casos y para algunos tipos de inversión, retornos incluso superiores a los obtenidos por la inversión privada.

\section{Resumen}

In this work we revise the main theoretic models on which the inquiry of the relationship between public investment and economic growth has been based, and also the empirical results obtained through its application. The empirical literature revised suggests, growingly, that such relationship exists, that it is positive and significant, and that public investment might have, in some cases and for some kinds of investment, even higher returns than those obtained by private investment.
\end{abstract}

Clasificación JEL: E62, F43

Palabras clave: Inversión pública, Crecimiento económico

* Centro de Estudios SocioEconómicos (CISE). Universidad Autónoma de Coahuila (UAdeC). Unidad Campo Redondo, edificio "S", C. P. 25280. Saltillo, Coahuila. Correo electrónico: grodriguez@cise.uadec.mx 


\section{Introducción}

Las últimas dos o tres décadas han presenciado una constante, y con frecuencia acelerada, traslación del interés de la política económica desde la idea keynesiana del fortalecimiento de la demanda agregada como instrumento para impulsar la economía, hacia un enfoque neoclásico que centra la atención en el lado de la oferta y, en particular, en la acumulación de los factores productivos como motor del crecimiento económico. ${ }^{1}$

Después de observar los resultados, menos que satisfactorios, arrojados por la política económica de menos gobierno (regulación y burocracia) y más mercado, seguida recientemente por algunos países, ${ }^{2}$ la cuestión de cual es el tamaño óptimo del sector gubernamental y de cual es el modo más eficiente de asignar el gasto público, adquiere notable relevancia. El debate académico ha producido una ingente serie de estudios y nuevos modelos con el fin de dar respuesta a estas preguntas y a otras más detalladas.

La motivación para realizar este trabajo surge de la noción de que el gasto público constituye el instrumento directo más importante con que el gobierno cuenta para influir sobre el crecimiento éconómico. ${ }^{3}$ Esta panorámica está organizada como sigue:

En la segunda sección se expone brevemente la evolución experimentada por la visión sobre el papel que al Estado le toca jugar para mejor contribuir al (o no interferir con el) óptimo desempeño de la economía. La sección tres propone, también brevemente, una distinción entre los diferentes tipos de gastos que el sector público puede realizar. Esta clasificación, tan arbitraria como cualquier otra, tiene el propósito de precisar el tipo de gasto al que se refieren las exposiciones subsiguientes.

La cuarta sección revisa la teoría que ha servido de marco para el análisis del gasto público y su influencia sobre el crecimiento, hasta donde sabemos, en la mayor parte de la literatura actual sobre el tema. Sin pretensiones de exhaustividad, se presentan algunos modelos que ilustran el modo en que se ha dado el análisis formal de la antedicha relación entre gasto público y crecimiento, así como sus principales conclusiones. La sección cinco, revisa una parte del trabajo empírico, principalmente el referido a la generación de hipótesis y modelos econométricos, dedicado a cuantificar (estimar) las diversas relaciones surgidas de dichas hipótesis, en particular las elasticidades de la productividad con respecto al gasto público en inversión. Valga decir que tampoco se trata de una revisión exhaustiva, pero que consideramos suficientemente representativa. La sección seis concluye con algunos comentarios finales.

1 El concepto de "crecimiento económico" aquí empleado se refere solamente al crecimiento de la productividad o del producto (promedio) per capita, ninguna cuestión referida al concepto de "desarrollo económico" se aborda.

2 Sólo para citar un ejemplo, en México el poder adquisitivo de los salarios mínimos ha experimentado un descenso sin precedentes en los últimos 15-20 años, y alrededor de un cuarto de la población total se encuentra en la pobreza extrema según cifras oficiales. Naturalmente, esto no ha impedido que la economía del país crezca en el sentido expresado en la nota anterior

3 Y de que, en una economía con gobiernos democráticamente electos, la política de gasto reflejará, en alguna medida, ciertas preferencias o criterios sociales. 


\section{El papel del estado en la economía: un poco de historia ${ }^{4}$}

Se atribuye al poeta francés Paul Valery la idea de que "si el Estado es fuerte, nos aplastará; si es débil, pereceremos". Presumiblemente, el estado ideal está entre estos dos extremos. Desde el punto de vista de la teoría económica y durante el último medio siglo principalmente, adquirieron notable preponderancia dos formas de analizar el papel del estado: el positivo y el normativo.

La economía normativa intenta definir qué es lo que el gobierno tendría que hacer para maximizar el bienestar; en tanto que la positiva describe y analiza lo que el gobierno efectivamente hace. ${ }^{5}$ En un mundo ideal ambas cosas se identificarían: el estado haría lo que se espera que haga y todas las reformas necesarias para maximizar el bienestar social se estarían llevando a cabo. En el mundo real, sin embargo. ambas cosas difieren y, con frecuencia, por mucho. Las razones son varias: diferencia de intereses entre gobierno y gobernados, errores y concepciones equivocadas por parte de los diseñadores de la política económica y efectos residuales de decisiones pasadas.

Usualmente, los economistas ponderan la participación del estado en la economía, a través de la medición de la recaudación y/o del gasto público como proporción del PIB. Según este criterio, la participación del estado en los países industrializados es, en promedio, dos veces mayor que en los países no desarrollados. Sin embargo, este criterio es parcial. En efecto, la influencia del estado, sobre la asignación de recursos en la economía y sobre la distribución, puede variar considerablemente con el nivel de las regulaciones que se impongan sobre mercados y agentes. Por otra parte, el estado también ha tenido una extensa participación en la producción directa de bienes y servicios.

Los economistas clásicos abogaban por una participación mínima del sector público: provisión de servicios públicos esenciales, manteniniento de la ley y del orden y defensa del país. Desde su punto de vista el gobierno debía garantizar los derechos de propiedad, la inviolabilidad de los contratos y proteger las libertades económicas y políticas de los individuos. Fstas son las llamadas actividades básicas del sector público. ${ }^{6}$ En parte debido a esto, durante el siglo XIX el papel del gobierno se mantuvo bastante limitado, el gasto público estaba en torno al 10\% del PIB y el laissez-faire era la filosofía económica dominante.

En cambio, el siglo XX presenció una gradual pero gran expansión del Estado: para los nuevos países industrializados el gasto pasó de $12 \%$ del PIB en 1913 a $45 \%$ en 1995 . Factores políticos y sociológicos contribuyeron a este cambio. El pensamiento Marxista y Socialista creó fuertes presiones para que los gobiernos jugarán un papel significativo en la redistribución del ingreso. El establecimiento del comunismo, en la Unión Soviética y luego en otros países, empujó a muchos estados hacia una economía "mixta". La distribución del ingreso se convirtió en un objetivo de política básico, que indujo altas tasas progresivas de gravación del ingreso, subsidios a bienes básicos, transferencias directas y un aumento del gasto público en salud y educación.

4 Esta sección se basa en Tanzi (1997).

5 En este trabajo empleamos indistintamente los términos Estado, Gobierno y Sector Público.

6 "Core activities". Smith (1778). 
El pensamiento keynesiano, por su parte, también presionó para que el gobierno sostuviera el ingreso disponible de los individuos durante las fluctuaciones cíclicas, con el objetivo de estabilizar la economía. Se creía que los países con sectores públicos grandes estaban menos sujetos a las fluctuaciones de los ciclos económicos.

Aunado al impacto de los pensamientos socialista y keynesiano, algunos "desarrollos técnicos" de la teoría económica después de la $2^{a}$ guerra mundial, dieron justificación adicional a la intervención pública. En particular, el concepto de bien público justificó la provisión de bienes y servicios con características de bien público, los cuales teóricamente serían subprovistos por el sector privado. Estrechamente relacionado, el concepto de externalidad también influyó: el gobierno tenía que intervenir para solucionar los fallos de mercado.

Por otra parte, la literatura temprana sobre desarrollo económico suponía con frecuencia que el gobierno tenía capacidades de las que carecía el sector privado: administrativas y de recursos e información principalmente. En los 50 's y 60 's, especialmente en los países en desarrollo se suponía, también frecuentemente, que el gobierno era el mejor juez para decidir que bienes eran "esenciales" o "necesarios" y cuales no.

En la actualidad, la experiencia ha mostrado que la visión idealista de cómo se lleva a cabo el diseño de la política económica y de cómo se implementa, está lejos de la realidad:

1) el sector público no es monolítico,

2) sus políticas pueden ser inconsistentes en el tiempo y en el espacio,

3) pueden ser influidas por el afán de lucro personal y por grupos de presión,

4) algunos de los que toman decisiones de política económica pueden ignorar el modo en que la economía realmente funciona,

5) puede haber problemas del tipo agente-principal,

6) las acciones pueden ser irreversibles,

7) la burocracia puede ser ineficiente o corrupta, o ambas.

La opinión de que el gobierno puede solucionar la mayoría de los problemas no es ya tan ampliamente aceptada como lo fue hace dos o tres décadas. En años recientes se ha notado que el crecimiento de la intervención gubernamental ha venido acompañado de un descuido de las actividades básicas del estado, arrojando como resultado un deterioro continuo en la calidad de los servicios básicos provistos por el gobierno: administración de justicia, cumplimiento de contratos, etc.

En consecuencia, la tendencia reciente es la de un estado menos interventor (de ahí las privatizaciones, desregulaciones, etc.). Reconociéndose, sin embargo, la necesidad de que el estado intervenga para mejorar el funcionamiento de los mercados en lugar de remplazarlos, en las actividades básicas y en la redistribución del ingreso.

\section{El crecimiento económico y el gasto público}

Como anotamos en la introducción, este trabajo se interesa por la relación entre el gasto público y el crecimiento económico. Sin embargo, esta relación puede ser extremadamente compleja merced a los distintos tipos de gasto que el 
sector público puede ejercer y a la diversa serie de respuestas que el crecimiento económico ofrece ante cada tipo de gasto. Esta sección tiene como propósito delimitar la parte de dicha relación que deseamos estudiar.

Podemos iniciar el análisis de las causas o fuentes del crecimiento dividiéndolas en dos: "causas próximas" y "causas últimas" (Hoffman, 2000). Las primeras se refieren a influencias inmediatas y medibles, las segundas a influencias de carácter institucional y cualitativo siendo por tanto más difícil su medición.

Maddison (1991) define las causas próximas del crecimiento como:"...those areas of causality where measures and models have been developed by economists and statisticians. Here the relative importance of different influences can be more readily assessed."

Con respecto a las causas últimas, y al papel que juega el gasto público en ellas, podemos decir que se trata de un área de estudio menos abordada por la investigación cuantitativa dada la dificultad, arriba anotada, de medir los efectos que pueden tener sobre el crecimiento económico.

Consideremos un aumento del gasto público en defensa: Por una parte este incremento podría tener un efecto negativo sobre el crecimiento, puesto que tales recursos podrían asignarse a actividades productivas. Pero por otra parte, el fortalecimiento de la defensa proporciona la seguridad necesaria para la inversión productiva. Análogamente, el gasto público dedicado al mantenimiento de los derechos de propiedad, al establecimiento de mecanismos para el cumplimiento de contratos y, al mejoramiento de la eficiencia administrativa, pueden tener por un lado efectos negativos sobre el crecimiento al desviar recursos de la parte productiva de la economía, pero por otro lado, establecen condiciones cuya ausencia obstaculizaría la fluidez y la eficiencia del sector productivo así como el adecuado funcionamiento de los mercados, pudiendo llegar al extremo de conducir al caos y paralizar la actividad económica por completo.

Este tipo de influencias es sin embargo difícil de medir y, al menos en parte, por eso no se ha desarrollado una literatura y modelos formales, que teórica y/o empíricamente evalúen cuantitativamente su contribución al crecimiento económico. El alcance de este trabajo no permite ahondar en esta parte de la relación entre el gasto público y el crecimiento, siendo su objetivo el de estudiar las causas próximas que el gasto público modifica y sus efectos sobre el crecimiento de la economía.

De acuerdo con lo anterior proponemos la siguiente caracterización del modo en que se distribuye el gasto público, con el objetivo de especificar la parte que estudiaremos:

1) Causas próximas: La parte efectivamente gastada (gasto en inversión y gasto corriente) en bienes y servicios que, en principio, puede afectar directamente la productividad del sector privado, a este tipo de gasto le llamaremos $\left(G_{p}\right)$.

2) Causas últimas: La parte efectivamente gastada (gasto en inversión y gasto corriente) que impacta directamente la demanda agregada, pero no la productividad privada (defensa, burocracia, administración de la justicia, legislación y otros)

3) La parte desviada por corrupción e ineficiencia administrativa. 
A su vez, $G_{p}$ puede dividirse en tres:

i) Inversión en infraestructuras (capital público): carreteras, redes de provisión de agua, de electricidad, comunicaciones, puentes, etc.

ii) Inversión en capital humano: educación, asistencia sanitaria, recreación y otros.

iii) Inversión en $I+D$ (incluyendo apoyos a la $I+D$ privada).

Luego de realizar esta caracterización sucinta de las causas del crecimiento y de los distintos destinos del gasto público, en lo que sigue analizaremos los efectos que el gasto público destinado a modificar las causas próximas, tiene sobre el crecimiento. Esto es. veremos como, la también llamada inversión pública (capital público, capital humano e $I+D$ ), afecta el desempeño de la economía, principalmente en el largo plazo.

\section{Aspectos teóricos}

El marco analítico actualmente utilizado en una gran parte de la investigación cuantitativa del crecimiento económico, consiste básicamente en la especificación de un modelo dinámico, que maximiza la utilidad presente de un individuo (o dinastía), sujeta a una restricción tecnológica; esto es, utilizando la notación estándar en la literatura:

$$
\max \int_{0}^{\infty} \exp (-\rho t) u(c(t)) N(t) \mathrm{d} t,
$$

tal que

$$
Y(t)=F(K(t), N(t)),
$$

y, a partir de la contabilidad, la regla de comportamiento intertenporal:

$$
\begin{aligned}
Y(t) & =C(t)+S(t) \\
& =C(t)+I_{B}(t)=C(t)+\delta K(t)+I_{N}(t), \\
& =C(t)+\delta K(t)+\mathrm{d} K(t) / \mathrm{d} t
\end{aligned}
$$

en donde:

$Y(t)$ es el producto en el momento $t$.

$C(t)$ es el consumo en el momento $t$.

$S(t)$ es el ahorro en el momento $t$.

$I_{B}(t)$ es la inversión bruta en el momento $t$.

$\delta$ es la tasa de depreciación que se supone constante.

$K(t)$ es el acervo de capital en el momento $t$.

$N(t)$ la población en el momento $t$.

$I_{N}(t)$ es la inversión neta en el momento $t$.

$\mathrm{d} K(t) / \mathrm{d} t$ es el cambio en el acervo de capital en el momento $t$.

Este modelo básico contiene una serie de supuestos y simplificaciones que hacen manejable su introducción (economía cerrada, un sólo sector, dos factores de producción, crecimiento poblacional exógeno, separabilidad aditiva de la 
función de utilidad, homogeneidad de la función de producción, etc.). La eliminación de una o varias de estas simplificaciones o supuestos, constituyen extensiones del modelo.

Una de las extensiones más importantes es la que relaja el supuesto de que la función de producción cumple las condiciones de Inada, específicamente, la de que los rendimientos marginales del capital son decrecientes. Esto da lugar a los "modelos de crecimiento endógeno", en donde es posible que la economía crezca sin especificar una tasa de crecimiento exógeno. El ejemplo más sencillo es el de la "tecnología $A K$ ", que cambia con respecto al modelo básico antes expuesto, solamente en la especificación de una función de producción de la forma: $Y=A K$. En donde $K$ se entiende como un "concepto amplio de capital".

Este marco analítico permite también analizar la relación de otras variables de la economía con el crecimiento: desarrollo del sistema financiero, inversión en capital humano, gasto en $I+D$, entre otros.

El interés de este trabajo se centra como ya dijimos, en el estudio de la influencia que el gasto gubernamental pueda tener en el crecimiento económico. Barro (1991) es uno de los primeros autores en presentar un modelo de crecimiento endógeno con gasto público, introduciéndolo en la función de produccción: $Y=A L^{1-\alpha} K^{\alpha} G^{1-\alpha}$. Con $L$ constante, el crecimiento de $G$ impide que los rendimientos marginales de $K$ sean decrecientes posibilitando así el crecimiento endógeno.

A partir del análisis de Barro, se ha profundizado el estudio de la relación entre el gasto público y el crecimiento económico, realizándose varias extensiones para analizar de manera más general y completa este fenómeno, como las expuestas por Sánchez-Robles (1995) y Barriga (1998), entre otros. En este contexto estudiamos la relación entre la inversión pública y el crecimiento económico.

\subsection{El gasto público que afecta directamente la productividad pri- vada}

\subsubsection{Gasto público y crecimiento}

A) El modelo de bienes públicos de los servicios gubernamentales productivos

De acuerdo con Barro y Sala-i-Martín (1995), los supuestos iniciales asumen que el gobierno compra una parte del producto privado y luego la utiliza para proveer servicios públicos gratuitos a productores privados, esto equivale a la condición de que la función de producción del gobierno no difiere de la función de producción de cada firma.

Si $G$ es el gasto gubernamental, suponiendo que $G$ es no-rival y no-excluyente, i.e. $G$ es un bien público, cada empresa utiliza $G$ en su totalidad sin que disminuya la cantidad de $G$ disponible para las demás, por ejemplo, el conocimiento generado por la investigación públicamente financiada. 
A.1) La Economía Descentralizada

Se supone, siguiendo la exposición de Barro y Sala-i-Martín (1995), que la función de producción de la empresa $i$ es

$$
Y_{i}=A L_{i}^{1-\alpha} K_{i}^{\alpha} G^{1-\alpha}, \quad 0<\alpha<1 .
$$

Esto es, presenta rendimientos constantes a escala en los insumos privados. Manteniendo la fuerza laboral agregada, $L$, constante, la economía también enfrentará rendimientos constantes a escala en $K_{i}$ y $G$, es decir, con $L$ constante, la función de producción será homogénea de grado uno en $K_{i}$ y $G$. Debido a esto la economía tiene la posibilidad de crecer "endógenamente", en efecto, el crecimiento de $G$ evitaría que, al acumularse, el capital llegue a presentar rendimientos marginales decrecientes. Dicho de otro modo, si la función de producción agregada es:

$$
Y=A L^{1-\alpha} K^{\alpha} G^{1-\alpha} .
$$

Entonces:

$$
\frac{\partial Y}{\partial K}=A L^{1-\alpha} \alpha \frac{G^{1-\alpha}}{K^{1-\alpha}}=P M g K>0
$$

$\mathrm{y}$

$$
\frac{\partial^{2} Y}{\partial K^{2}}=-A L^{1-\alpha} \alpha(1-\alpha) \frac{G^{1-\alpha}}{K^{2-\alpha}}<0 .
$$

Por lo que, si $G$ aumenta con $K$, el capital mantendrá su rendimiento marginal, posibilitando así el crecimiento endógeno.

Nótese que la forma de la función de producción implica que los servicios públicos son complementarios con los insumos privados en el sentido de que un incremento en $G$ aumenta los productos marginales de $L_{i}$ y de $K_{i}$. También, si el exponente de $G$ fuese menor que $1-\alpha$, tendríamos rendimientos decrecientes en $K_{i}$ y $G$, y no sería posible el crecimiento endógeno. Si dicho exponente fuese mayor, las tasas de crecimiento tenderían a crecer. Por tanto, el modelo se concentra en el caso en que el exponente de $G$ es exactamente igual a $1-\alpha$.

Se supone que el gobierno mantiene un presupuesto balanceado que financia con un impuesto proporcional constante $\tau$ (no lump-sum) sobre el producto bruto agregado, de modo que

$$
G=\tau Y,
$$

en donde el cociente $G / Y$ es constante.

La función de utilidades de cada empresa $i$, es:

$$
\begin{aligned}
\Pi_{i} & =(1-\tau) A L_{i}^{1-\alpha} K_{i}^{\alpha} G^{1-\alpha}-w L_{i}-(r+\delta) K_{i} \\
& =(1-\tau) Y_{i}-w L_{i}-(r+\delta) K_{i}(=0 \text { en competencia perfecta }),
\end{aligned}
$$

donde $w$ es la tasa salarial (salario real en unidades de producto por unidad de $L)$ y $(r+\delta)$ la tasa de arrendamiento del capital. Y la contribución marginal del capital está dada por:

$$
\frac{\partial \Pi_{i}}{\partial K_{i}}=(1-\tau) \frac{\partial Y_{i}}{\partial K_{i}}-(r+\delta)=0,
$$


de donde:

$$
(1-\tau) \alpha A L_{i}^{1-\alpha} K_{i}^{\alpha-1} G^{1-\alpha}=(r+\delta)
$$

y con $k_{i}=K_{i} / L_{i}=K / L=k$ tenemos:

$$
(r+\delta)=(1-\tau) \alpha A G^{1-\alpha} k^{\alpha-1} .
$$

De (4) y (8) se obtiene para $G$ :

$$
\begin{aligned}
& G=\tau Y=\tau A L^{1-\alpha} K^{\alpha} G^{1-\alpha} \Rightarrow G^{\alpha}=\tau A L k^{\alpha} \\
& G=(\tau A L)^{1 / \alpha} k .
\end{aligned}
$$

Sustituyendo ahora (13) en (12):

$$
\begin{aligned}
(r+\delta) & =(1-\tau) \alpha A k^{\alpha-1}\left[(\tau A L)^{1 / \alpha} k\right]^{1-\alpha}=(1-\tau) \alpha A^{1 / \alpha}(\tau L)^{(1-\alpha) / \alpha} \\
r & =(1-\tau) \alpha A^{1 / \alpha}(\tau L)^{(1-\alpha) / \alpha}-\delta
\end{aligned}
$$

Puesto que $\tau$ y $L$ son constantes, (14) implica que $r$ no varía con $k$. Por su parte, el consumidor representativo resuelve el siguiente problema:

$$
\begin{gathered}
\max U=\int_{0}^{\infty} e^{-\rho t} \frac{c_{t}^{1-\theta}-1}{1-\theta} \mathrm{d} t, \\
\text { s.a. } w_{t}+r_{t} a_{t}=c_{t}+\dot{a_{t}} .
\end{gathered}
$$

El Hamiltoniano puede establecerse como:

$$
H=e^{-\rho t}\left[\left(c_{t}^{1-\theta}-1\right) /(1-\theta)+\lambda_{t}\left(w_{t}+a_{t} r_{t}-c_{t}\right)\right], \text { con } \mu_{t}=\lambda_{t} e^{-\rho t},
$$

y las condiciones de primer orden:

$$
\begin{aligned}
& \frac{\partial H}{\partial c_{t}}=e^{-\rho t}\left(c_{t}^{-\theta}-\lambda_{t}\right)=0, \\
& \frac{\partial H}{\partial a}=e^{-\rho t}(\lambda t) r_{t}=-\frac{\partial \mu_{t}}{\partial t} .
\end{aligned}
$$

Implican:

$$
\mathrm{d} c / \mathrm{d} t=\gamma_{c}=\left(r_{t}-\rho\right) / \theta .
$$

La condición de transversalidad es:

$$
\lim _{t \rightarrow \infty} a_{t} \mu_{t}=0
$$

de donde, considerando (20):

$$
c_{t}=c_{0} e^{(r t-\rho) / \theta}
$$


con $c_{0}$ aún por determinar:

$$
\lim _{t \rightarrow \infty} a_{t} c_{0}^{-\theta} e^{-r t}=0
$$

Del problema de optimización que resuelve la empresa, conocemos $r$, por lo que:

$$
\gamma_{c}=\left[(1-\tau) \alpha A^{1 / \alpha}(\tau L)^{(1-\alpha) / \alpha}-\delta-\rho\right] / \theta .
$$

Como en este caso $a_{t}=k_{t}=K_{t}$, entonces $\dot{a}_{t} / a_{t}=\gamma_{a}=\left(w_{t} / a_{t}\right)+r_{t}-\left(c_{t} / a_{t}\right)$, y por tanto $\gamma_{k}=\gamma_{c}$. En conclusión, el gasto público afecta al crecimiento a través de dos canales:

i) El término $(1-\tau)$ representa el efecto negativo del impuesto sobre el producto marginal (después de impuestos) del capital, y

ii) El término $\tau^{(1-\alpha) / \alpha}$ es el efecto positivo de los servicios públicos sobre el producto marginal.

Con la condición usual de primer orden se obtiene el "tamaño" óptimo del gobierno que maximiza la tasa de crecimiento de la economía:

$$
\frac{d \gamma}{d \tau}=0=\frac{\alpha A^{1 / \alpha} L^{(1-\alpha) / \alpha}\left(\frac{1-\alpha}{\alpha}\right) \tau^{(1-2 \alpha) / \alpha}-\alpha A^{1 / \alpha} L^{(1-\alpha) / \alpha}\left(\frac{1}{\alpha}\right) \tau^{(1-\alpha) / \alpha}}{\theta} .
$$

Así:

$$
\tau=G / Y=1-\alpha .
$$

Este resultado se interpreta notando que, a partir de $Y_{i}=A L_{i}^{1-\alpha} K_{i}^{\alpha} G^{1-\alpha}$

$$
\frac{\partial Y}{\partial G}=(1-\alpha) \frac{Y}{G}=\frac{1-\alpha}{\tau}=1
$$

debido a que el producto marginal social de $G$ es la suma de su producto marginal en cada empresa.

La condición $\tau=1-\alpha$ corresponde a la condición natural de eficiencia para el tamaño del gobierno, i.e. iguala el beneficio social de $G$ a su costo social, que es uno.

En este caso, es posible verificar la optimalidad paretiana de este resultado analizando la versión centralizada de la economía. El planificador social elige las sendas temporales de $G(t)$ y $c(t)$ que resuelven el siguiente problema (para simplificar hay solamente un consumidor y una empresa):

$$
\begin{gathered}
\max U=\int_{0}^{\infty} e^{-\rho t} \frac{C_{t}^{1-\theta}}{1-\theta} \mathrm{d} t, \\
\text { s.a } Y_{t}=A K_{t}^{\alpha} G_{t}^{1-\alpha}=C_{t}+G_{t}+\mathrm{d} K_{t} / \mathrm{d} t+\delta K .
\end{gathered}
$$

Del mismo modo que antes:

$$
H=e^{-\rho t}\left[\left(C_{t}^{1-\theta}-1\right) /(1-\theta)+\lambda_{t}\left(A K_{t}^{\alpha} G^{1-\alpha}-C-G-\delta K\right)\right], \mu_{t}=\lambda_{t} e^{-\rho t},
$$




$$
\frac{\partial H}{\partial C_{t}}=e^{-\rho t}\left(C_{t}^{-\theta}-\lambda_{t}\right)=0,
$$

de donde $\lambda_{t}=C_{t}^{-\theta}$, y por lo tanto:

$$
\begin{gathered}
\mathrm{d} \lambda_{t} / \mathrm{d} t=-\theta C_{t}^{-\theta}\left(\mathrm{d} C_{t} / \mathrm{d} t\right) / C_{t}, \\
\frac{\partial H}{\partial G_{t}}=e^{-\rho t} \lambda_{t}\left(A K_{t}^{\alpha}(1-\alpha) G_{t}^{-\alpha}-1\right)=0,
\end{gathered}
$$

de donde $\mathrm{d} Y / \mathrm{d} G=1$ y también $(1-\alpha)=G_{t} / Y_{t}$. Finalmente:

$$
\frac{\partial H}{\partial K_{t}}=-\dot{\mu}=e^{-\rho t} \lambda_{t}\left(\alpha A K_{t}^{\alpha-1} G_{t}^{1-\alpha}-\delta\right)
$$

de donde $\mu_{t}=\lambda_{t} e^{-\rho t} \Rightarrow \mathrm{d} \mu_{t} / \mathrm{d} t=e^{-\rho t}\left(\mathrm{~d} \lambda_{t} / \mathrm{d} t-\rho \lambda_{t}\right)$

$$
\begin{gathered}
\lambda_{t}\left(\alpha A K_{t}^{\alpha-1} G^{1-\alpha}-\delta\right)=\rho \lambda_{t}-\mathrm{d} \lambda_{t} / \mathrm{d} t=C_{t}^{-\theta} \rho+\theta C_{t}^{-\theta}\left(\mathrm{d} C_{t} / \mathrm{d} t\right) / C_{t} \\
\theta\left(\mathrm{d} C_{t} / \mathrm{d} t\right) / C_{t}=\alpha A K_{t}^{\alpha-1} G^{1-\alpha}-\delta-\rho \Rightarrow \gamma_{c}=1 / \theta\left(\alpha A K_{t}^{\alpha-1} G^{1-\alpha}-\delta-\rho\right) \\
\gamma_{c}=1 / \theta(\mathrm{d} Y / \mathrm{d} K-\delta-\rho) .
\end{gathered}
$$

Que es semejante a la tasa de crecimiento obtenida en la economía descentralizada, excepto por el término $(1-\tau)$. La interpretación es directa: el equilibrio de mercado no alcanza el óptimo paretiano debido a que cada uno de los productores considera la productividad marginal del capital después de impuestos, es decir, el impuesto genera una brecha entre la productividad marginal del capital privada y social y, al ser menor la privada, la economía descentralizada conduce a un resultado ineficiente.

El modelo hasta aquí analizado, predice efectos de escala debido a que los servicios gubernamentales son bienes públicos que pueden extenderse sin costo a más usuarios, por tanto, si $L$ aumenta, la función de producción presentará rendimientos crecientes en virtud de que $\alpha+(1-\alpha)+(1-\alpha)>1$, lo cual implica tasas de crecimiento per capita crecientes, de modo que, para estudiar los estados estacionarios, es necesario suponer tasas nulas de crecimiento poblacional.

El hecho de que no se detecten efectos de escala más importantes, puede significar que los servicios públicos se congestionan, esto es, la cantidad de $G$ disponible para cada empresa disminuye a medida que otras lo utilizan. EI siguiente desarrollo teórico explora esta posibilidad.

B) El modelo de congestión de los servicios públicos

El mismo trabajo de Barro y Sala-i-Martín (1995), expone este modelo en donde se supone que los servicios estatales susceptibles de congestionarse son, por ejemplo, las carreteras, la provisión de agua, policía y bomberos, administración de justicia y otros. Para iniciar el análisis se considera la siguiente función de producción:

$$
Y_{i}=A K_{i} f(G / Y), \quad f^{\prime}>0, f^{\prime \prime}<0
$$


En donde un incremento de $G$ con respecto al producto agregado, $Y$, aumentará $Y_{i}$ para un $K_{i}$ dado. Esta formulación supone que $G$ tiene que aumentar con respecto a $Y$ si es que han de aumentar los servicios públicos disponibles para cada uno de los usuarios.

De acuerdo con lo anterior, dada la razón $G / Y$, cada empresa tiene rendimientos constantes con respecto a $K_{i}$, si $G$ crece a la misma tasa que $Y, G / Y$ permanecerá fija y la economía tendrá la posibilidad de generar un crecimiento endógeno, como en el modelo $A K$.

Se supone aquí también que $\tau=G / Y$ ( $G$ crece a la misma tasa que $Y$ por definición), por tanto, el producto marginal del capital, después de impuestos, es:

$$
(1-\tau) \mathrm{d} Y / \mathrm{d} K_{i}=(1-\tau) A f(\tau)=r+\delta .
$$

Aplicando el mismo procedimiento utilizado para el modelo anterior:

$$
\gamma_{c}=(1 / \theta)(r-\rho)
$$

que es la misma tasa de crecimiento de $k$ y de $y$, capital e ingreso per cápita respectivamente. Combinando ambas expresiones se obtiene:

$$
\gamma=(1 / \theta)[A(1-\tau) f(\tau)-\delta-\rho] .
$$

Como $f^{\prime}>0$ y $f$ " $<0$, se puede obtener nuevamente el "tamaño" óptimo del gobierno, $\tau=G / Y$, definido como aquél que maximiza la tasa de crecimiento $\gamma$. En efecto, igualando a cero la derivada parcial de $\gamma$ con respecto a $\tau$ :

$$
(1-\tau) f^{\prime}(\tau)-f(\tau)=0, \text { y por tanto }:(1-\tau) f^{\prime}(\tau)=f(\tau) .
$$

Aplicando el teorema de la función implícita, y como $Y=A K f(G / Y)$, entonces:

$$
\frac{\partial Y}{\partial G}=-\frac{\frac{\partial F}{\partial G}}{\frac{\partial F}{\partial Y}}=-\frac{A K f^{\prime}(G / Y)(1 / Y)}{A K f^{\prime}(G / Y)\left(-G / Y^{2}\right)-1}=\frac{f^{\prime}(\tau)}{f(\tau)+\tau f^{\prime}(\tau)}
$$

Sustituyendo por la condición de maximización $f(\tau)=(1-\tau) f^{\prime}(\tau)$ se obtiene:

$$
\frac{\partial Y}{\partial G}=\frac{f^{\prime}(\tau)}{f^{\prime}(\tau)-\tau f^{\prime}(\tau)+\tau f^{\prime}(\tau)}=1
$$

que es exactamente igual al costo marginal del gasto gubernamental.

La resolución del problema de optimización del planificador social proporciona el mismo resultado que el obtenido por la economía descentralizada, independientemente de la forma funcional de $f(\cdot)$, el resultado más notable es, por tanto, que a diferencia del anterior modelo, aquí se obtiene la eficiencia paretiana con un impuesto proporcional y, de hecho, el impuesto de suma fija sería ineficiente: los productores individuales no tomarían en cuenta el impacto negativo, sobre la productividad, de un aumento en el producto. 


\subsubsection{Dinámica y transición al estado estacionario}

Hasta aquí, los modelos analizados no presentan dinámica transicional alguna porque se trata de modelos del tipo $A K$, Sánchez-Robles (1995) desarrolla una generalización que permite estudiar los aspectos dinámicos de la transición al estado estacionario.

Con los supuestos estándar y por simplicidad:

$$
\begin{gathered}
\max U(0)=\int_{0}^{\infty} e^{-\rho t} \frac{c_{t}^{1-\theta}-1}{1-\theta} \mathrm{d} t, \\
\text { s.a } Y=A \Psi\left\{(b K)^{\Psi}[(1-b) G]^{\Psi}\right\}^{1 / \Psi}, 0<\Psi<1, \\
\mathrm{~d} K / \mathrm{d} t=(1-\tau) Y-c-\delta K, \\
\mathrm{~d} G / \mathrm{d} t=\tau Y-\delta G .
\end{gathered}
$$

El trabajo se normaliza constantemente a uno, $K$ es el acervo de capital privado y $G$ el acervo de capital público. $G$ puede interpretarse como un bien rival de provisión pública, sujeto a congestión y a excluibilidad completa; las empresas no toman en cuenta su contribución marginal al incremento de la congestión. $A, b$ y $\Psi$ son parámetros tecnológicos; la elasticidad de sustitución entre los insumos es $1 /(1-\Psi)$.

La función de producción tiene rendimientos constantes a escala y la productividad marginal de cada factor es decreciente. La violación de una de las condiciones de Inada, la de que la productividad marginal de ambos tipos de capital tiende a cero, posibilita el crecimiento endógeno.

La solución del planificador social, estableciendo el respectivo Hamiltoniano, aplicando las condiciones de primer orden y la de transversalidad, permite caracterizar la tasa de crecimiento de la economía en el estado estacionario:

$$
\left(\frac{\mathrm{d} c}{\mathrm{~d} t}\right)\left(\frac{1}{c}\right)=\gamma_{c}=\left(\frac{1}{\theta}\right)\left[A\left[b^{\Psi}+(1-b)^{\Psi}\left(\frac{1-b}{b}\right)^{\Psi /(1-\Psi)}\right]^{(1-\Psi) / \Psi} b^{\Psi}-r-\delta\right] .
$$

En el estado estacionario todas las variables relevantes crecen a la misma tasa; el consumo crece si la productividad marginal del capital, neta de depreciación, excede la tasa de preferencia temporal. Cuanto mayor sea la elasticidad de sustitución, mayor será la respuesta de los agentes a la diferencia entre la tasa de retorno neta y la tasa de preferencia temporal.

El cociente (capital privado/capital público) es: $K / G=b /(1-b)^{1 /(1-\Psi)}$. Intuitivamente, el hecho de que el planificador pueda elegir el tipo impositivo óptimo, ocasiona que en el estado estacionario las productividades marginales de ambos tipos de capital sean iguales.

Para el análisis descentralizado, se nota que, en el contexto del presente modelo existe algo parecido a una externalidad: cuando un individuo destina una unidad adicional a la inversión, ocasiona un incremento del capital público vía elevación de renta e ingresos impositivos lo que a su vez hace más productivo el capital privado; y puesto que este efecto externo es pequeño, el individuo no lo tendrá en cuenta al tomar sus decisiones de inversión. Asumiendo la existencia 
del equilibrio competitivo, lo anterior implica que la tasa de inversión y por consiguiente de crecimiento, será menor en el equilibrio competitivo que en la solución del planificador, i.e., ineficiente en el sentido de Pareto.

Para obtener la solución descentralizada se supone que los agentes siguen una regla de decisión óptima similar a la del planificador social, de manera que la razón $K / G$ es igual a la obtenida en la solución centralizada. Aplicando el método de optimización usual y sustituyendo la razón $K / G$ por el valor antes obtenido, el resultado es:

$$
\begin{aligned}
\left(\frac{\mathrm{d} c}{\mathrm{~d} t}\right)\left(\frac{1}{c}\right) & =\gamma_{c} \\
& =\left(\frac{1}{\theta}\right)\left((1-t) A\left(\left[b^{\Psi}+(1-b)^{\Psi}\left(\frac{1-b}{b}\right)^{\frac{\Psi}{(1-\Psi)}}\right]^{\frac{(1-\Psi)}{\Psi}} b^{\Psi}\right)-\rho-\delta\right),
\end{aligned}
$$

de donde, la tasa de crecimiento del equilibrio competitivo es menor que la tasa alcanzada en la economía centralizada.

Con respecto al análisis de la transición dinámica hacia el estado estacionario, como el sistema puede resolverse sólo por métodos numéricos, SánchezRobles (1995) emplea el método de eliminación de tiempos, diseñado por Mulligan y Sala-i-Martin $(1991,1993)$, para realizar simulaciones y estudiar las trayectorias temporales de las principales variables.

En particular, verifica las siguientes características de la transición:

i) La tasa de crecimiento del consumo es cada vez menor conforme la economía se aproxima al estado estacionario, en concordancia con la idea de la convergencia condicional.

ii) Cuanto mayor es la elasticidad de sustitución entre los insumos, la transición es más lenta, pero menos traumática: la variabilidad de las tasas de crecimiento a lo largo de la transición decrece.

iii) Las funciones de producción del tipo CES representan un caso intermedio entre los modelos neoclásicos (transición lenta al estado estacionario) y los del tipo " $A K$ " (transición inmediata al estado estacionario).

Hasta aquí, uno de los resultados principales que hemos encontrado, se refiere al tamaño óptimo del gobierno. Este tamaño es función directa de un parámetro tecnológico, $\tau=1-a$, lo cual no resulta sorprendente, puesto que lo hemos introducido como un insumo más de la función de producción y, naturalmente, el proceso de maximización de ésta, arroja un tamaño óptimo para aquél.

Por tanto, es importante mantener presente que estos resultados son válidos en la medida, y sólo en la medida, en que el gasto gubernamental bajo análisis, afecte la productividad del modo planteado por estos modelos. La determinación del tamaño óptimo del gobierno en un sentido más amplio que incluya por ejemplo el efecto a través de la demanda agregada y del multiplicador, requiere, obviamente, de un análisis más completo y por ende, más sofisticado y de difícil tratamiento. 


\subsubsection{Una economía con dos sectores}

Con la finalidad de verificar si existe evidencia empírica que apoye la intervención del Estado en la economía mexicana y si el gasto público es útil como instrumento de política para suavizar el ciclo económico, Barriga (1995) realiza la estimación econométrica de dos modelos empíricos.

El primero de ellos, dirigido a estimar si la intervención pública en México genera una externalidad (positiva o negativa) en el resto de la economía, se desarrolla a partir de los trabajos de Rati Ram (1986) y Gershon Feder (1983).

Se supone que la economía está compuesta por el sector público $(G)$ y el privado $(C)$, el producto en cada uno de ellos depende del trabajo $(L)$ y del capital $(K)$, se supone también que el producto (tamaño) del gobierno genera una externalidad sobre el producto del sector privado, por lo que las funciones de producción pueden escribirse como:

$$
\begin{gathered}
C=C\left(K^{C}, L^{C}, G\right), \\
G=G\left(K^{G}, L^{G}\right) .
\end{gathered}
$$

Los insumos totales están dados por:

$$
\begin{gathered}
L^{C}+L^{G}=L, \\
K^{C}+K^{G}=K .
\end{gathered}
$$

El producto total $(Y)$ es la suma del producto de ambos sectores:

$$
C+G=Y \text {. }
$$

$\mathrm{Si}$

$$
G_{K} / C_{K}=G_{L} / C_{K}=1+\delta,
$$

en donde los subíndices denotan la derivada parcial de la función de producción con respecto al insumo de que se trate, entonces el signo de $\delta$ indica que sector tiene una mayor productividad marginal.

Diferenciando (48) y (49):

$$
\begin{gathered}
\mathrm{d} C=\left(C_{K}^{*} I_{C}\right)+\left(C_{L}^{*} \mathrm{~d} L^{C}\right)+\left(C_{G} * \mathrm{~d} G\right), \\
\mathrm{d} G=\left(G_{K} * I_{G}\right)+\left(G_{L} * \mathrm{~d} L^{G}\right),
\end{gathered}
$$

en donde $\mathrm{d} K=I$.

De (52) el diferencial total es:

$$
\mathrm{d} Y=\mathrm{d} C+\mathrm{d} G .
$$

Sustituyendo (54) y (55) en (56), y combinando con el diferencial de productividad factorial intersectorial (53):

$$
\mathrm{d} Y=C_{K} I_{C}+C_{L} \mathrm{~d} L^{C}+C_{G} \mathrm{~d} G+(1+\delta) C_{K} I_{G}+(1+\delta) C_{L} \mathrm{~d} L^{C},
$$




$$
\mathrm{d} Y=C_{K}\left(I_{N}+I_{G}\right)+C_{L}\left(\mathrm{~d} L^{C}+\mathrm{d} L^{G}\right)+C_{G} \mathrm{~d} G+\delta\left(C_{K} I_{G}+C_{L} \mathrm{~d} L^{G}\right) .
$$

De (55) y (53):

$$
\frac{\mathrm{d} G}{1+\delta}=\frac{\left(G_{K} * I_{G}\right)+\left(G_{L} * \mathrm{~d} L^{G}\right)}{1+\delta}=C_{K} I_{G}+C_{L} \mathrm{~d} L^{G}
$$

Y como, por otro lado, $I=I_{C}+I_{G}$ y $\mathrm{d} L=\mathrm{d} L^{C}+\mathrm{d} L^{G}$. Sustituyendo en $\left(57^{\prime}\right)$ :

$$
\mathrm{d} Y=C_{K} I+C_{L} \mathrm{~d} L+\left(\delta /(1+\delta)+C_{G}\right) \mathrm{d} G .
$$

Suponiendo una relación lineal entre la productividad marginal del trabajo y el producto medio por trabajador, $C_{L}=\beta(Y / L)$.

Dividiendo (59) por $Y$, y haciendo $C_{K}=\alpha$, se obtiene la ecuación de crecimiento agregado:

$$
\gamma_{Y}=\alpha(I / Y)+\beta \gamma_{L}+[\delta /(1+\delta)-\theta] \gamma_{G}(G / Y)+\theta \gamma_{G} .
$$

O definiendo $\delta^{\prime}=\delta /(1+\delta)$.

$$
\gamma_{Y}=\alpha(I / Y)+\beta \gamma_{L}+\left(\delta^{\prime}-\theta\right) \gamma_{G}(G / Y)+\theta \gamma_{G},
$$

donde:

$\gamma$ representa la tasa de crecimiento de la variable correspondiente,

$\beta$ es la elasticidad del producto privado con respecto a $L$,

$\alpha$ es el producto marginal de $K$ en el sector $C$,

$\theta=C_{G}(G / C)$ es la elasticidad del producto privado con respecto a $G$,

$I$ es la inversión y se supone igual a $\mathrm{d} K$.

Econométricamente, si $\theta$ se interpreta como un parámetro constante a lo largo de la muestra, (60) puede utilizarse para obtener estimados de $\delta$ y $\theta$ que indicarán, respectivamente, la diferencia intersectorial en cuanto a la productividad de los factores y el efecto marginal de la externalidad del producto del gobierno en el resto de la economía.

Un caso especial a destacar es que, si $\delta^{\prime}=\theta,\left(60^{\prime}\right)$ se reduce a:

$$
\gamma_{Y}=\alpha(I / Y)+\beta \gamma_{L}+\theta \gamma_{G} .
$$

Como vemos en $\left(60^{\circ}\right), \theta$ da sólo el efecto de externalidad del tamaño del gobierno y no el efecto total. Sin embargo, como (61) está basada en la restricción $\delta^{\prime}$ $=\theta$, el estimado de $\theta$ da un estimado de $\delta^{\prime}$ (y de $\delta$ ), y por tanto el efecto total siempre que se cumpla dicha restricción.

Si se postula que $C_{G}$, en lugar de $\theta$, es el parámetro constante, entonces (60) se reescribe como:

$$
\gamma_{Y}=\alpha(I / Y)+\beta \gamma_{L}+\left[\delta^{\prime}+C_{G}\right] \gamma_{G}(G / Y) .
$$


La ventaja de estimar (62), sobre (60) y (59), es que se obtiene el efecto total de la externalidad del coeficiente de $\gamma_{G}(G / Y)$, pero no es posibe obtener por separado los efectos de la externalidad y el diferencial de la productividad factorial.

Otro punto a destacar es que mientras en (60) la colinealidad entre $\gamma_{G}$ y $\gamma_{G}(G / Y)$ puede acarrear problemas, (61) y (62) no tienen ese inconveniente. Comparando estas dos últimas, aunque simple e informativa, (61) está sujeta a la igualdad entre $\delta^{\prime}$ y $\theta$. Por otro lado, aunque menos informativa en el sentido de que no podemos obtener por separado los estimados de $\delta$ y $C_{G},(62)$ no está sujeta a restricciones paramétricas.

\subsection{El gasto público y los derechos de propiedad}

Como dijimos antes, una parte del gasto público puede ser dedicada a actividades que no afectan directamente la productividad del sector privado, pero que si pueden tener un efecto indirecto.

Específicamente, servicios públicos como los facilitados por la policía, los juzgados, la defensa nacional y similares, pueden interpretarse como servicios que afectan la probabilidad de que los individuos mantengan sus derechos sobre los bienes que poseen y por tanto tengan incentivos para acumular capital y propiciar el crecimiento económico.

Suponiendo que la probabilidad $p$ de que un individuo mantenga la propiedad sobre su producto es una función creciente de $G / Y$, esto es:

$$
p=p(G / Y), \quad p^{\prime}>0, \quad p^{\prime \prime}<0 .
$$

Por ejemplo, si $G$ es el gasto agregado en policía, la cantidad de protección que obtiene cada individuo depende de la razón $G / Y$, i.e., de la cantidad de producto que la policía tenga que vigilar.

Suponiendo que los productores se interesan solamente por la tasa de retorno esperada, entonces la condición de maximización de utilidad (después de impuestos) puede expresarse como:

$$
(1-\tau) A p(\tau)=r+\delta
$$

y la tasa de crecimiento estará dada por:

$$
\gamma=(1 / \theta)[A(1-\tau) p(\tau)-\delta-\rho]
$$

Los resultados son similares a los anteriores y las mismas conclusiones valen. Las principales predicciones empíricas de esta clase de modelos pueden resumirse en la Gráfica 1.

La relación predicha entre la tasa de crecimiento y la participación de los servicios en el PIB, $G / Y$, no es monótona: la tasa de crecimiento aumenta con $G / Y$ cuando el gobierno es pequeño, pero declina cuando $G / Y$ se hace demasiado grande. 


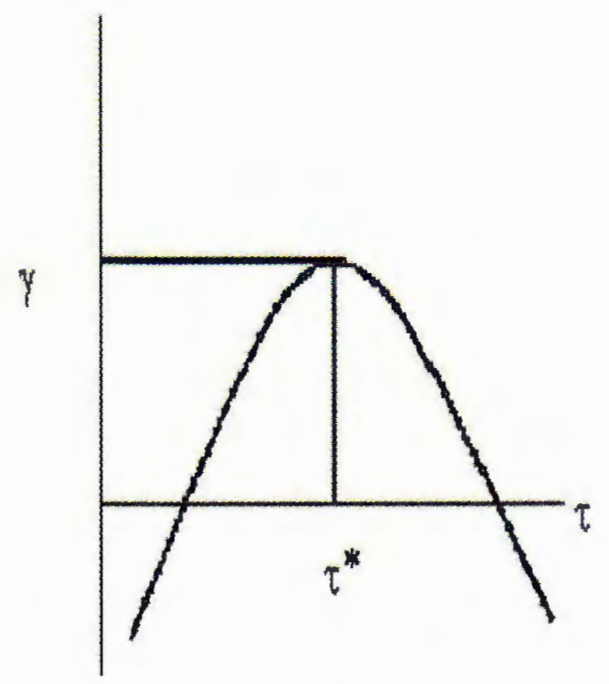

Gráfica 1.

\section{Resultados empíricos}

\subsection{I +D y progreso técnico}

El proceso de creación y difusión de nuevas tecnologías juega un papel importante en el crecimiento económico. El capital tecnológico presenta importantes elementos de bien público que dificultan la apropiación privada de su valor económico, reduciendo su rentabilidad privada por debajo de la social y dando lugar, en ausencia de medidas correctivas, a una tendencia a la sub-inversión en este tipo de capital en una economía de mercado. Existen por tanto, razones para la intervención pública en esta área. El hecho de que, por un lado, la investigación genera externalidades positivas, y por otro, puede haber externalidades negativas (por ejemplo la carrera por la patente), hace necesaria la intervención pública para corregir los fallos de mercado resultantes.

La teoría económica identifica el esfuerzo tecnológico como un factor importante para el crecimiento y sugiere que éste podría verse obstaculizado por fallos de mercado, que requerirían una intervención pública con vistas a conseguir un resultado eficiente. La evidencia empírica disponible, en líneas generales, tiende a confirmar estas conclusiones (IAE (1994)).

Es importante considerar también el efecto de los gastos en importación de tecnología. Estudios empíricos muestran que las actividades de generación y adopción de nuevas técnicas y productos pueden ser complementarias (Mansfield (1961)).

\subsection{Educación, productividad y crecimiento}

La educación es uno de los componentes más importantes de la inversión y uno de los determinantes centrales de la productividad de la fuerza laboral. 
El control de la oferta educativa es, por tanto, uno de los instrumentos más directos de que disponen los gobiernos para incidir sobre el crecimiento.

La evidencia empírica, en su conjunto, es consistente con la hipótesis de que el nivel de formación de la fuerza laboral es uno de los determinantes más importantes de su productividad y su capacidad para absorber el progreso técnico (IAE, 1994). Igual que antes, se dan fallos de mercado (externalidades positivas y negativas) que justifican la intervención pública (Landau (1983, 1986) y Barro (1991))

\subsection{Infraestructuras (capital público) y productividad}

La incidencia de las infraestructuras sobre la productividad ha recibido bastante atención en la literatura reciente. Gran parte de los trabajos se concentran en la estimación de funciones de producción donde el capital público aparece como argumento junto con el capital privado y el trabajo.

La serie de investigaciones desarrolladas a partir del trabajo de Aschauer (1989) coinciden en que los coeficientes del capital público son en general significativos. Sin embargo, la cuestión aún no puede darse por zanjada, véase por ejemplo a Argimón et al. (1993), Ford y Poret (1991), García-Milà et al. (1993), Holtz-Eakin (1992), Mas et al. (1993), Munell (1990, 1992), Munell y Cook (1990) y Serra y García-Fontes (1993).

En este sentido, vale la pena mencionar el trabajo sobre Portugal realizado por Lighthart (2000), quien analiza los resultados obtenidos por distintos autores sobre la elasticidad-producto del capital público (ver tabla 1).

La mayoría de los estudios de series de tiempo utilizan una función de producción Cobb-Douglas para estimar los efectos del capital público sobre el producto. En promedio, estos estudios estiman una elasticidad-producto del capital público $(\beta)$ de 0.25 para algunos países de la OECD cuando la función de producción se estima en niveles. Los estimados de $\beta$ varían considerablemente entre los distintos países pero están en el intervalo $0.20-0.30$ a un nivel del $95 \%$ de confianza. Si el modelo se estima en primeras diferencias los estimados de $\beta$ son, en promedio, más altos y los intervalos de confianza más amplios. Los estudios de panel -basados en datos regionales para un sólo país- encuentran en general coeficientes estimados mucho más bajos, lo cual puede atribuirse a "fugas" que reflejan el hecho de que, a nivel regional, no todos los efectos benéficos de derrame de la inversión pública pueden internalizarse.

Tabla 1: Estudios empíricos: El enfoque de la función de producción

\begin{tabular}{|c|c|c|c|c|}
\hline AUTOR & PAIS & $\beta$ & ESPECIFICACIÓN & DATOS \\
\hline Ratner (1983) & U.S. & 0.06 & CD, LL & TS, 1949-73 \\
\hline Aschauer (1989) & U.S. & 0.39 & CD, LL & TS, 1949-85 \\
\hline Ram and Ramsey (1989) & U.S. & 0.24 & CD, LL & TS, 1948-85 \\
\hline Munell (1990) & U.S. & 0.31 & CD, LL & TS, 1949-87 \\
& & $0.371 /$ & CD, LL & \\
\hline Aaron (1990) & U.S. & 0.41 & CD, LL & TS, 1952-85 \\
& & 0.27 & CD, DL & \\
\hline
\end{tabular}


Tabla 1: Estudios empíricos: El enfoque de la función de producción (continuación)

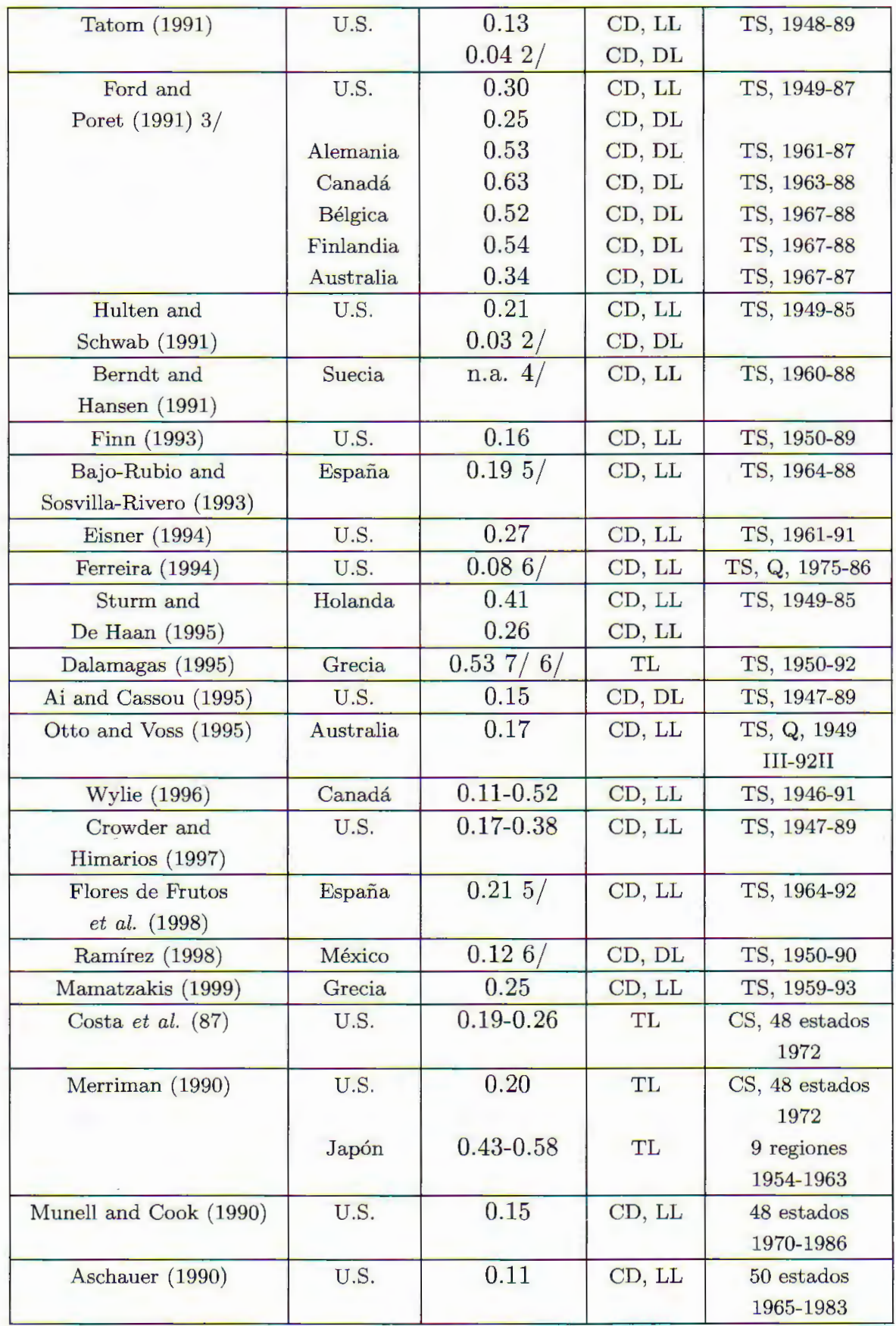


Tabla 1: Estudios empíricos: El enfoque de la función de producción (continuación)

\begin{tabular}{|c|c|c|c|c|}
\hline Eisner (1991) & U.S. & $0.172 /$ & $\mathrm{CD}, \mathrm{LL}$ & $\begin{array}{l}48 \text { estados } \\
1970-1986\end{array}$ \\
\hline $\begin{array}{c}\text { García-Milà } \\
\text { and McGuire (1992) }\end{array}$ & U.S. & $0.04-0.05$ & $\begin{array}{c}\mathrm{CD}+\mathrm{TL} \\
\mathrm{LL}\end{array}$ & $\begin{array}{l}48 \text { estados } \\
1969-1983 \\
\end{array}$ \\
\hline Munell (1993) & U.S. & $0.14-0.17$ & $\mathrm{CD}, \mathrm{LL}$ & $\begin{array}{l}48 \text { estados } \\
1970-1986 \\
\end{array}$ \\
\hline $\begin{array}{l}\text { Evans and Karras } \\
\text { (1994) }\end{array}$ & U.S. & n.a. 2/ & $\begin{array}{l}\mathrm{CD}, \mathrm{TL} \\
\mathrm{LL}, \mathrm{DL}\end{array}$ & $\begin{array}{l}48 \text { estados } \\
1970-1986\end{array}$ \\
\hline Holtz-Eakin (1992) & U.S. & n.a. 2/ & $\mathrm{CD}, \mathrm{LL}$ & $\begin{array}{l}48 \text { estados } \\
1976-1986\end{array}$ \\
\hline Pinnoi (1994) & U.S. & 0.08 & TL & $\begin{array}{l}48 \text { estados } \\
1970-1986 \\
\end{array}$ \\
\hline $\begin{array}{c}\text { Baltagi and } \\
\text { Pinnoi (1995) }\end{array}$ & U.S. & n.a. 2/ & $\mathrm{CD}, \mathrm{LL}$ & $\begin{array}{l}48 \text { estados } \\
1970-1986\end{array}$ \\
\hline $\begin{array}{c}\text { Mas et al. } \\
(1996)\end{array}$ & España & 0.08 & $\mathrm{CD}, \mathrm{LL}$ & $\begin{array}{c}17 \text { regiones } \\
1980-1989\end{array}$ \\
\hline $\begin{array}{l}\text { García-Milà et al. } \\
\text { (1996) }\end{array}$ & U.S. & n.a. $2 /$ & $\mathrm{CD}, \mathrm{DL}$ & $\begin{array}{l}48 \text { estados } \\
1970-1983 \\
\end{array}$ \\
\hline
\end{tabular}

Fuente: Lighthart (2000). Clave: $C D=$ Cobb-Doglas, LL $=$ Estimado en niveles $\log , \mathrm{DL}=$ Estimado en primeras diferencias $\log , \mathrm{TL}=$ Translog en niveles, TS $=$ Serie de tiempo, $\mathrm{CS}=$ corte transversal, $\mathrm{P}=$ Datos de Panel y $Q=$ Datos Trimestrales.

1/ Sin restricciones sobre la función de producción.

2/ El coeficiente es insignificante al $5 \%$.

3/ Estudio de 11 países de la OECD. Sólo los coeficientes de los países listados fueron significativos.

4/ Encuentra valores implausibles de los coeficientes.

5/ Relación de cointegración identificada.

6/ Inversión pública en lugar de acervo de capital público.

7/ Sólo cuando el déficit fiscal es incluído en la ecuación, de otro modo el coeficiente es insignificante.

\section{Comentarios Finales}

En este trabajo hemos estudiado la relación de un componente específico del gasto público (inversión pública) con el crecimiento económico. Esto es, hemos visto como el gasto público, que modifica las causas próximas, afecta el crecimiento de la economía.

A nivel teórico resalta la cuestión de la optimalidad: dado que la inversión pública tiene que financiarse ¿cuál es el tamaño óptimo de la inversión? La respuesta, naturalmente, cumple la regla usual de optimización: el costo social deberá igualar el beneficio social. En este sentido, el resultado más relevante 
es el de la necesidad de la inversión pública: si bien a partir de cierto nivel el incremento de la inversión tendría efectos negativos, también es cierto que su anulación sería negativa, por tanto la economía requiere de ese nivel de inversión pública para maximizar su crecimiento, ya sea por la corrección de fallos de mercado, por efectos de derrame, por complementariedad u otros.

En el terreno empírico se trata de probar si efectivamente existe la relación positiva entre inversión pública y crecimiento, y de cuantificar esta relación para distintos tipos de inversión. Esto resulta de interés para el diseño de la política económica, pues es necesario determinar la distribución de los recursos a invertir. A diferencia del análisis teórico, no se analiza empíricamente el financiamiento de la inversión, más bién, la cuestión de interés es: Dado el monto de recursos a invertir (por ejemplo, exógenamente determinado, como los fondos de integración destinados por la Unión Europea a países como Portugal) ¿Cuál es la mejor manera de distribuirlos entre los distintos tipos de inversión disponibles para lograr el más alto impacto sobre el crecimiento económico?

La literatura empírica revisada sugiere, crecientemente, que la mencionada relación existe y que puede tener, en algunos casos y para algunos tipos de inversión, retornos incluso superiores a los obtenidos por la inversión privada. Sin embargo, y en parte debido a su propia naturaleza estas investigaciones no son concluyentes. Como quiera, en la realidad las economías nacionales siempre destinan recursos a la inversión pública, lo más indicado sería estudiar sus particularidades y tratar de determinar las áreas en que la inversión puede ser más productiva y el modo en que la distribución de los recursos a invertir daría los resultados globales de mayor deseabilidad en el mediano y largo plazo.

Tal vez valga también la pena reflexionar sobre el carácter parcial y las limitaciones de los resultados antes expuestos. En cuanto al carácter parcial de estos enfoques, el análisis cubre aspectos principalmente cuantitativos con el fin de medir el impacto efectivo del gasto público sobre el crecimiento; enfoques que no consideran el efecto del gasto a través de la demanda, entre otros. Por otro lado, el análisis teórico está guiado por el concepto paretiano de eficiencia, lo cual en principio, conduce los resultados en direcciones claramente predefinidas (preservación del stau qou) ignorando posibilidades alternativas. En particular, se ignora el papel redistributivo que el gobierno actualmente juega en prácticamente todas las economías del mundo, papel como antes vimos, que surge, en principio, a partir de la presión social generada por las difíciles situaciones distributivas surgidas a partir del laissez faire, y que actualmente forman parte de un concepto ya tradicional, comúnmente aceptado y con frecuencia oficial. La siguiente cita se ha tomado del Tomo I de las Cuentas por Sectores Institucionales 1993-1998, del Sistema de Cuentas Nacionales de México, publicado por el Instituto Nacional de Estadística Geografía e Informática (INEGI):

"Los objetivos, funciones y comportamiento económico del gobierno general son totalmente distintos a los de los demás sectores, pues organiza y financia el suministro de bienes no de mercado, incluidos los servicios individuales y colectivos prestados a los hogares y a la comunidad, ocupándose también de la distribución y redistribución del ingreso y la riqueza mediante los impuestos y transferencias".

Este tipo de gasto gubernamental es ajeno a la lógica de la eficiencia paretiana y entra en consideraciones que rebasan el análisis meramente cuantitativo 
y que, a final de cuentas, exigen la introducción de juicios de valor y/o metas definidas por procesos político-sociales cuando de implementar el gasto gubernamental se trata.

\section{Bibliografía}

Argimón, I. et al. (1993). Productividad e infraestructuras en la economía española. Banco de España, Documento de Trabajo, No. 9313.

Aschauer, D. (1989). Is Public Expenditure Productive? Journal of Monetary Economics, 23.

Bajo, O. R. (1998). Integración regional, crecimiento y convergencia: un panorama. Revista de Economía Aplicada, 16.

Barriga, E. D. (1995). Crecimiento económico y gobierno. El caso de México. El Trimestre Económico.

Barro, R. (1991). Economic Growth in a Cross Section of Countries. Quarterly Journal of Economics.

Barro, R. and X. Sala-i-Martín (1995). Economic Growth. McGraw Hill.

De la Fuente, A. (1996). Economía regional desde una perspectiva neoclásica. De convergencia y otras historias. Revista de Economía Aplicada, 10.

De la Fuente, A. (1994). Crecimiento y convergencia: un panorama selectivo de la evidencia empírica. Cuadernos Económicos de ICE, 58.

De la Fuente, A. (1993). Expenditure Levels and Incentives in Public R\&D. Los efectos del mercado único en el desarrollo regional y su incidencia sobre la planificación a largo plazo en las regiones del objetivo, 1(3), IAE.

De la Fuente, A. y J. M. da Rocha, (1993). Capital humano, productividad y crecimiento. Los efectos del mercado único en el desarrollo regional y su incidencia sobre la planificación a largo plazo en las regiones del objetivo, 1(2), IAE.

Domar, E. (1946). Capital Expansion, Rate of Growth and Employment. Econometrica, 14. Ermisch, J.F, and W. G. Huff (1998). Hypergrowth in an East Asian NIC: Public Policy and Capital Accumulation in Singapure. World Development, 27(1).

Ford, R. and P. Poret (1991). Infrastructure and Private-Sector Productivity. OECD. Ecconomic Studies, 17.

García-Milà et al. (1993). The Effect of Public Capital in State-Level Production Functions Reconsidered. Mimeo, Feb.

Harrod, R. F. (1939). An Essay in Dynamic Theory. Economic Journal, 49.

Hoffman, A. A. (2000). The Economic Development of Latin America in the Twentieth Century. Edward Elgar.

Holtz-Eakin, D. (1992). Public Sector Capital and the Productivity Puzzle. NBER, Working Papers, No 4122.

Instituto de Análisis Económico (IAE) (1994). Crecimiento y convergencia regional en España y Europa. Campus Universidad Autónoma de Barcelona.

Kaldor, N. and J.A. Mirrlees (1961). A new Model of Economic Growth. Review of Economic Studies, 29.

Kahn, R. F. (1959). Exercises in the Analysis of Growth. Oxford-Economic Papers. New series, 11.

Landau, D. (1983). Government Expenditure and Economic Growth: A Cross Country Study. Southern Economic Journal. Jan.

Landau, D. (1986) Government and Economic Growth in the Less Developed Countries: An Empirical Study for 1960-80. Economic Development and Cultural Change. Oct.

Lichtenberg, F. (1993). R\&D Investment and International Productivity Differences. NBER. Working Paper No. 4161.

Lighthart, J. (2000). Public Capital and Output Growth in Portugal: An Empirical Analysis. IMF, Working Paper, WP/00/11.

Lucas, R. (1988). On the Mechanics of Economic Development. Journal of Monetary Economics, 22. 
Mankiw, G et al. (1992). A Contribution to the Empirics of Economic Growth. Quarterly Journal of Economics.

Mansfield, E. (1961). Technical Change and the Rate of Imitation. Econométrica, 4.

Mas, M. et al. (1993). Capital público y productividad de la economía Española. Mimeo, IVIE.

Munell, A. (1992). Infrastructure Investment and Economic Growth. Journal of Economic Perspectives, 6(4).

Munell, A. (1990). Why has Productivity Declined? Productivity and Public Investment. New England Economic Review. Federal Reserve Bank of Boston, Jan./Feb.

Munell, A. and L. Cook (1990). How Does Public Infrastructure Affect Regional Economic Performance? New England Economic Review. Federal Reserve Bank of Boston, Sept./Oct.

Muñiz, I. O. (1998). Externalidades, localización y crecimiento: una revisión bibliográfica. Estudios Regionales, 52.

Phelps, E. S. (1965). A critique of Neutralism. In Fiscal Neutrality toward Economic Growth, McGraw-Hill.

Rao, G. (1998). Accomodating Public Expenditure Policies: the Case of Fast Growing Asian Economies. World Development, 26(4).

Robinson, J. (1962). A Model of Accumulation. In Essays in the Theory of Economic Growth, Macmillan.

Sánchez-Robles, B. (1995). Capital público y crecimiento económico: Un modelo alternativo. Cuadernos de Economía, 23.

Sen, A. (1970), Growth Economics. Penguin Books.

Serra, D. y W. García-Fontes (1993). Capital público, infraestructura y crecimiento. Los efectos del mercado único en el desarrollo regional y su incidencia sobre la planificación a largo plazo en las regiones del objetivo, 1(2), IAE.

Smith, A. (1789). An Inquiry into the Nature and Causes of the Wealth of Nations.

Solow, R. M. (1956). A contribution to the Theory of Economic Growth. Quarterly Journal of Economics, 70 .

Tanzi, V. (1997). The Changing Role of the State in the Economy: A Historical Perspective. IMF, Working Paper, WP/97/114. 